\title{
Formação do enfermeiro na perspectiva do cuidado integral e trabalho em equipe
}

\author{
Nurse training from the perspective of integral care and teamwork \\ La formación de enfermeras desde la perspectiva del cuidado integral y el trabajo en equipo
}

Recebido: 07/01/2021 | Revisado: 08/01/2021 | Aceito: 09/01/2021 | Publicado: 10/01/2021

Fernanda Costa Martins Gallotti

ORCID: https://orcid.org/0000-0002-9063-1273

Universidade Federal de Sergipe, Brasil

E-mail: fercosmart@gmail.com

Lorena Emanuelle Rodrigues Dos Santos

ORCID: https://orcid.org/0000-0003-1488-8832

Universidade Tiradentes, Brasil.

E-mail: lorenarodrigues100@ hotmail.com

Vanessa Gomes Alves Dias

ORCID: https://orcid.org/0000-0002-7793-4608 Universidade Tiradentes, Brasil

E-mail: danvanart@hotmail.com

Queila Samara dos Santos Farias

ORCID: https://orcid.org/0000-0002-4686-4539 Universidade Tiradentes, Brasil

E-mail: queilasamara20@gmail.com

Manuela de Carvalho Vieira Martins

ORCID: https://orcid.org/0000-0003-1222-5955

Universidade Tiradentes, Brasil

E-mail: manuela.cvm@hotmail.com

Rebecca Maria Oliveira de Góis

ORCID: https://orcid.org/0000-0002-3935-5904

Universidade Estadual de Feira de Santana, Brasil

E-mail: rebecca.gois@ hotmail.com

Maria Pureza Ramos de Santa Rosa

ORCID: https://orcid.org/0000-0003-4835-3043

Universidade Tiradentes, Brasil

E-mail: maria_pureza@unit.br

Rodrigo Gallotti Lima

ORCID: https://orcid.org/0000-0002-0786-7358

Instituto Federal de Sergipe, Brasil

E-mail: rodrigo.lima@ifs.edu.br

Lenilson Santos da Trindade

ORCID: https://orcid.org/0000-0002-4114-3740

Universidade Tiradentes, Brasil

E-mail: lenilsontrindade@msn.com

Mairim Russo Serafini

ORCID: https://orcid.org/0000-0003-4223-3470

Universidade Federal de Sergipe, Brasil.

E-mail: maiserafini@hotmail.com

\begin{abstract}
Resumo
Prestar um cuidado integral é importante para que o paciente tenha suas necessidades atendidas em diferentes ângulos. Este estudo objetivou analisar a percepção dos acadêmicos sobre a integralidade do cuidado e trabalho em equipe no processo de sua formação profissional. Trata-se de um estudo exploratório, descritivo, com abordagem quantitativa de corte transversal. Participaram da pesquisa 191 estudantes do curso de enfermagem de uma universidade privada de Aracaju do sexto ao décimo período. Com predomínio de respostas do sexo feminino, as respostas evidenciaram a importância de iniciar atividades que estimulem o trabalho em equipe e a integralidade no cuidado ainda durante a formação profissional. É de extrema importância a inserção do trabalho em equipe e do cuidado integral nas grades curriculares, sendo considerado o primeiro passo para conseguir uma assistência de qualidade em todos os sistemas de saúde.
\end{abstract}

Palavras-chave: Integralidade em saúde; Equipe de assistência ao paciente; Educação em enfermagem.

\begin{abstract}
Providing comprehensive care is important for the patient to have his needs met at different angles. This study aimed to analyze the students' perception of the integrality of care and teamwork in the process of their professional training. This is an exploratory, descriptive study, with a quantitative cross-sectional approach. 191 nursing students from a
\end{abstract}


private university in Aracaju participated in the research from the sixth to the tenth period. With a predominance of female responses, the responses showed the importance of starting activities that encourage teamwork and comprehensive care even during professional training. It is extremely important to include team work and comprehensive care in the curriculum, being considered the first step towards achieving quality assistance in all health systems.

Keywords: Integrality in health; Patient assistance team; Education nursing.

\section{Resumen}

Brindar una atención integral es importante para que el paciente satisfaga sus necesidades desde diferentes ángulos. Este estudio tuvo como objetivo analizar la percepción de los estudiantes sobre la integralidad del cuidado y el trabajo en equipo en el proceso de su formación profesional. Se trata de un estudio exploratorio, descriptivo, con un enfoque cuantitativo transversal. 191 estudiantes de enfermería de una universidad privada de Aracaju participaron de la investigación del sexto al décimo período. Con predominio de respuestas femeninas, las respuestas mostraron la importancia de iniciar actividades que fomenten el trabajo en equipo y la atención integral incluso durante la formación profesional. Es de suma importancia incluir el trabajo en equipo y la atención integral en el plan de estudios, siendo considerado el primer paso para lograr una asistencia de calidad en todos los sistemas de salud.

Palabras clave: Integralidad en salud; Equipo de asistencia al paciente; Educación en enfermería.

\section{Introdução}

A atuação do enfermeiro perpassa o cuidado integral, em seus múltiplos sentidos, sendo componente essencial no sistema de saúde diante dos últimos acontecimentos no contexto da saúde mundial e vem conquistando reconhecimento e promovendo debates e novas pesquisas (Reuter, Santos \& Ramos, 2018; Santos et al., 2019).

A enfermagem é compreendida como uma ciência de natureza humanística, que busca enxergar o indivíduo de maneira holística, inter-relacionando fatores que possam interferir no processo de adoecimento. Dessa forma, através da assistência, o profissional consegue abordar o paciente de forma que a integralidade seja alcançada, proporcionando a ampliação do cuidado (Azevedo et al., 2019).

Hidelgard Peplau em sua teoria das relações interpessoais descreve etapas de trabalho interligadas e muitas vezes sobrepostas para a interação dos enfermeiros com os pacientes (Deane \& Fain, 2015). Essa teoria foi a primeira da enfermagem a valorizar a importância das relações interpessoais no processo saúde-doença, trazendo uma ideia de que o enfermeiro deve evoluir junto ao paciente através da escuta qualificada fazendo com que o mesmo expresse suas necessidades e assim o profissional possa desenvolver um ambiente mais favorável e um raciocínio clínico sobre o quadro do paciente (Pinheiro et al., 2019).

No contexto do cuidado integral as relações interpessoais perpassam a relação enfermeiro - paciente, pela necessidade do olhar ampliado e intervenção conjunta de toda equipe de enfermagem, tal como, da equipe multiprofissional. Barros e Ellery (2016) afirmam que através da interação entre as equipes, torna-se possível o desenvolvimento de habilidades que perpetuem no processo saúde-doença, além de potencializar o fortalecimento do sistema de saúde.

$\mathrm{O}$ trabalho em equipe fundamenta-se nas relações interpessoais, na rotina diária, nas experiências vivenciadas e compartilhadas, respeitando a particularidade e individualidade de cada membro, para que na junção desses diferentes seres, o denominador comum seja a prestação de uma assistência de qualidade ao paciente. Não deixando de lado os princípios éticos e a morais que cada um traz consigo (Barros \& Ellery, 2016).

Trabalhar em equipe, no contexto da saúde, é uma atividade complexa, pois é comum haver desigualdades interprofissionais no ambiente de trabalho. No entanto, é importante que exista interação e comunicação entre as diferentes profissões com um objetivo único, facilitando a tomada de decisões, favorecendo o usuário e assim prestando uma atenção integral (Peduzzi et al., 2020).

Integralidade do cuidado e trabalho em equipe estão interligados e dependentes, para que ambos ocorram é necessária uma cooperação entre a equipe, união de saberes e objetivos claros, dessa forma, os pacientes serão avaliados holisticamente e 
observados obedecendo a equidade, mesmo com as diferentes especificidades (Chaves et al., 2017).

A academia em enfermagem tem um papel importante no processo da integralidade, promovendo uma formação que estimule a autonomia profissional permitindo que o estudante vivencie a prática de trabalhar em equipe desde a graduação, preparando-os para atuar nos diversos níveis de complexidade de forma que consiga alcançar o cuidado integral através do trabalho em equipe (Barbosa et al., 2019).

Diante disso, delineou-se como questão da pesquisa: Como os acadêmicos de enfermagem percebem a integralidade do cuidado e o trabalho em equipe na sua formação profissional?

Nesse contexto, propõe-se através desta pesquisa, analisar a percepção dos acadêmicos sobre a integralidade do cuidado e trabalho em equipe no processo de sua formação profissional. A discussão deste tema torna-se relevante, pois, partindo do pressuposto que, a perspectiva dos estudantes pode operar como um indicador do processo formativo e da inserção profissional, permitindo comparações, ajustes e mudanças curriculares quando forem pertinentes contribuindo para melhor desenvolvimento do processo de trabalho e reflexão sobre a integralidade do cuidado.

\section{Metodologia}

\section{Tipo de Estudo}

Trata-se de um estudo exploratório, de cunho descritivo, com abordagem quantitativa de corte transversal. Optou-se por este tipo de estudo por apresentar objetividade na coleta de dados e tratar-se de um estudo rápido, sendo útil no planejamento educacional e levantamento de questões (Pereira et al., 2018).

\section{Local e Participantes do Estudo}

A população do estudo foi composta por 191 estudantes do Curso de Graduação em Enfermagem de uma Instituição de Ensino Superior do estado de Sergipe. Foram incluídos na pesquisa estudantes que regularmente matriculados do sexto ao décimo semestre do curso e que aceitaram participar da pesquisa. A escolha dos semestres se deu por contemplar práticas clínicas e estágio curricular supervisionado. Foram excluídos os estudantes que realizaram preenchimento inadequado do instrumento de coleta de dados.

\section{Coleta de Dados}

A coleta de dados ocorreu entre abril e maio de 2020. Para realização da coleta utilizou-se uma adaptação do questionário Readiness Inter professional Learning Scale - RIPLS, na versão portuguesa traduzida e validada por Peduzzi e Normann (2013).

A versão em português da RIPLS aborda três dimensões "Trabalho em equipe e colaboração", "Identidade profissional" e "Atenção à saúde centrada no paciente”, distribuídos em 27 afirmativas. Em análise ao PPC do curso no qual os discentes estão inseridos e de forma a alcançar os objetivos propostos nessa pesquisa foram utilizadas 21 afirmativas do RILPS e acrescido mais duas dimensões "Competências profissionais" e "Percepção na formação", totalizando 31 afirmativas.

O questionário foi disponibilizado em formato eletrônico, contendo informações, relevância e objetivos do estudo. Mediante a concordância do Termo de Consentimento Livre e Esclarecido (TCLE), as seções do formulário eram disponibilizadas. 


\section{Procedimentos de análise dos dados}

A tabulação e organização do banco de dados ocorreu por meio do programa Excel Profissional Plus 2019 e, em seguida, analisado estatisticamente pelo software Statistical Package for the Social Sciences (SPSS) 25,0, onde foram obtidos resultados em frequência absoluta e relativa. O intervalo de confiança utilizado foi padronizado em 95\% para apresentar significância estatística $(\mathrm{p}<0,05)$.

Em relação aos questionamentos dos itens RIPLS, utilizou-se o teste Qui-quadrado de Pearson e o teste Exato de Fisher para avaliar a diferença da distribuição dos dados entre o "sexo", "matriculado em disciplina com ensino clínico ou estágio curricular supervisionado", "realização de estágio extracurricular voltado para assistência de enfermagem", "atuação como monitor em disciplina específica de enfermagem com ensino clínico" e "semestre alocado em 2020.1".

\section{Procedimentos Éticos}

Conforme o que pressupõe as diretrizes éticas que respaldam a pesquisa com seres humanos, o estudo seguiu o que dispõe código de ética dos profissionais de enfermagem e a Resolução 466/12 do Conselho Nacional de Saúde, obtendo parecer favorável após apreciação do Comitê de Ética em Pesquisa sob com parecer 1.867.828. A pesquisa envolve riscos mínimos onde os sujeitos envolvidos terão sigilo assegurado pelos pesquisadores e não passarão por nenhum tipo de constrangimento já que a entrevista será individualizada e sem identificação.

\section{Resultados}

A taxa de resposta dos questionários correspondeu 31,5\% dos estudantes, considerando o total de alunos matriculados nos semestres avaliados. A maioria era de mulheres (78,5\%), com idade entre 19 e 46 anos (77\% até 24 anos), cursando o último semestre do curso 25,1\% (Figura 1).

Durante a graduação, os discentes podem participar de diversas atividades que possibilitam aperfeiçoar habilidades aplicadas no cuidado integral e ampliam a interação em equipe. Dos entrevistados, 24,1\% referem ter experiência com estágio extracurricular direcionado à assistência de enfermagem e 12,6\% desenvolveram atividades de monitoria em disciplinas específicas de enfermagem. 
Figura 1 - Características da amostra do estudo $(\mathrm{n}=191)$.
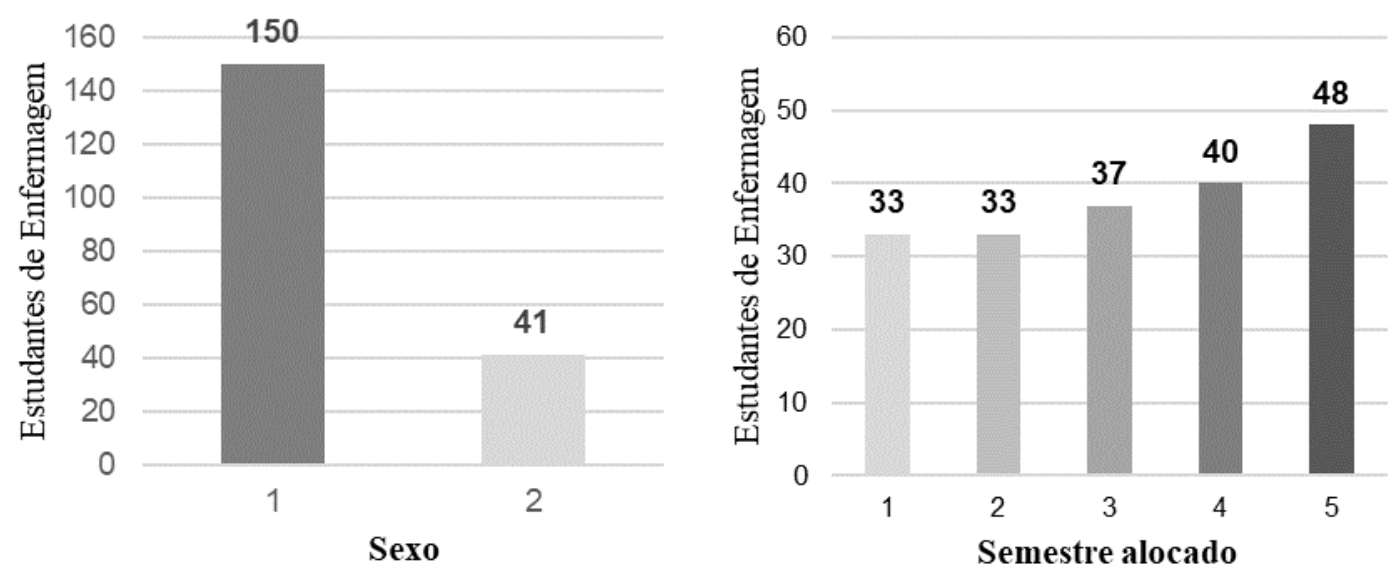

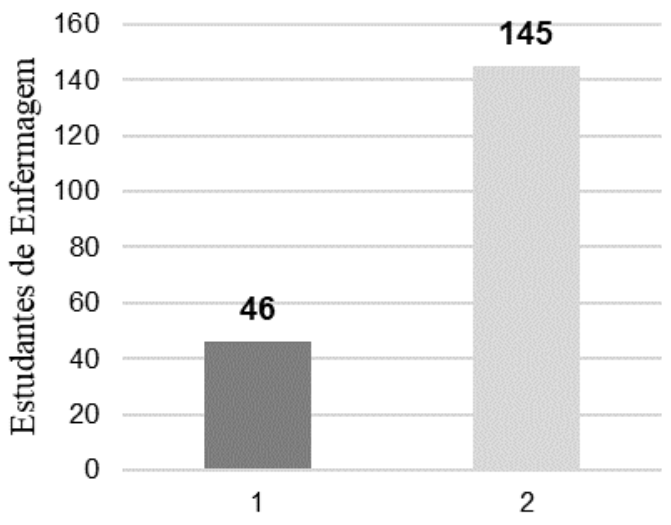

Estágio Extracurricular

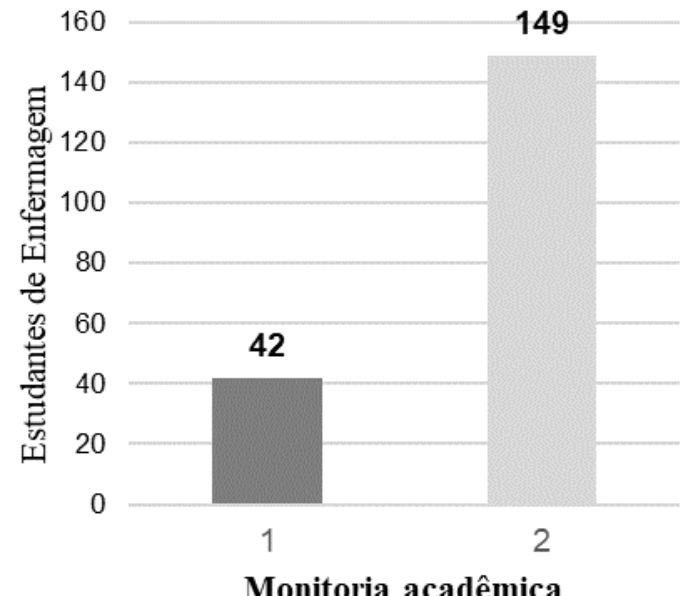

Monitoria acadêmica

Fonte: Autores (2020).

Quando se analisam os fatores do questionário aplicado, destacam-se algumas assertivas que tiveram uma concordância alta (acima de 80\%) sobre a disponibilidade para trabalho em equipe, tais como: "Para que a aprendizagem em pequenos grupos funcione, os estudantes precisam confiar e respeitar uns aos outros"; "Habilidades de trabalho em equipe são essenciais na aprendizagem de todos os estudantes da área da saúde”. Essas assertivas apresentaram significância estatística $(\mathrm{p}<0,05)$ em relação ao semestre dos alunos, observa-se que a concordância aumenta quanto mais avançados os alunos estão na graduação.

$\mathrm{Na}$ análise estatística exploratória verificou-se que os discentes, diante das afirmativas, possuem atitudes e prontidão semelhantes entre si, contudo, na comparação das variáveis sexo, estágio extracurricular, monitoria apresentam diferenças significativas (Tabela 1). As assertivas 11, 12, 21 e 26 apresentaram diferença significativa ao menos em duas das análises realizadas. Em A11 observa-se que a maioria dos discentes $(95,2 \%)$ que já tiveram vivências com monitorias discordam da assertiva que aponta que a função dos profissionais de saúde é apoiar aos médicos. Nota-se dessa forma que os mesmos percebem que cada profissional tem sua atribuição e importância dentro da equipe. Dos alunos que não tiveram experiências em monitoria $8,7 \%$ concordam da assertiva e $8,1 \%$ tem posicionamento neutro. Na afirmativa 12, "Preciso adquirir muito mais conhecimento e habilidades que estudantes de outras profissões da saúde" observa-se divergência entre as respostas, no qual, 
os alunos com vivências em monitorias e estágio extracurricular se colocam indiferentes a assertiva. No entanto, é importante o conhecimento aplicado em cada profissão, como também, usá-lo de forma interdisciplinar.

$\mathrm{Na}$ assertiva 21 mostra que os estudantes do sexo feminino e os que referem estágio extracurricular tem uma visão positiva quanto à atribuição do enfermeiro quase em sua totalidade. A parte científica é essencial para o cuidado, portanto, deve haver uma interação emocional com o paciente simultaneamente, valorizando-o. No tocante a A26 que retrata sobre gerenciamento e administração tanto da força de trabalho, dos recursos físicos e materiais e de informação, os discentes com relato de monitoria apontam em quase sua totalidade as contribuições da graduação nessa esfera.

Tabela 1. Distribuição das respostas dos estudantes de Enfermagem nos itens da RIPLS. Sergipe, 2020.

\section{Porcentagem de resposta por ponto da Escala Likert}

\section{Sexo X Fatores/itens RIPLS}

\section{Fator 1. Trabalho em equipe e colaboração}

7. Considerando minha graduação, não desperdiçaria meu tempo aprendendo junto com estudantes de outras profissões da saúde. ${ }^{\mathrm{A}}$

\begin{tabular}{|c|c|c|c|c|c|c|}
\hline Masculino & $33(80,5)$ & $8(19,5)$ & 0 & 0 & 0 & 60001 \\
\hline Feminino & $63(42)$ & $63(42)$ & $6(4)$ & $14(9,3)$ & $4(2,7)$ & $<0,001$ \\
\hline
\end{tabular}

9. Gostaria de ter a oportunidade de trabalhar em projetos, em pequenos grupos, com estudantes de outras profissões da saúde. $^{\mathrm{A}}$

\begin{tabular}{lllclll}
\hline Masculino & 0 & 0 & 0 & $12(29,3)$ & $29(70,7)$ \\
\hline Feminino & 0 & 0 & $6(4)$ & $70(46,7)$ & $74(49,3)$ & $<0,036$
\end{tabular}

11. A função dos demais profissionais da saúde é principalmente apoio aos médicos ${ }^{\mathrm{A}}$

\begin{tabular}{lcccccc}
\hline Masculino & $30(73,2)$ & $6(14,6)$ & $1(2,4)$ & 0 & $4(9,8)$ & \multirow{2}{*}{$<0,001$} \\
\hline Feminino & $73(48,7)$ & $55(36,7)$ & $13(8,6)$ & $9(6)$ & 0 & \\
\hline
\end{tabular}

\section{Fator 3. Atenção à saúde centrada no paciente}

21. Na minha profissão são necessárias habilidades de interação e cooperação com os pacientes (situação do paciente). ${ }^{\mathrm{A}}$

\begin{tabular}{lllllll}
\hline Masculino & 0 & 0 & 0 & $21(51,2)$ & $20(48,8)$ & \multirow{2}{*}{$<0,026$}
\end{tabular}

\section{Faz ou fez estágio extracurricular voltado para assistência de enfermagem X Fatores/itens RIPLS} Fator 2. Identidade profissional

12. Preciso adquirir muito mais conhecimento e habilidades que estudantes de outras profissões da saúde. ${ }^{\mathrm{A}}$

\begin{tabular}{lcccccc}
\hline Sim & $5(10,9)$ & $9(19,6)$ & $23(50)$ & $9(19,5)$ & 0 \\
\hline Não & $13(9)$ & $64(44,1)$ & $32(22,1)$ & $16(11)$ & $20(13,8)$ & $<0,001$ \\
\hline
\end{tabular}

16. Minha principal responsabilidade como profissional é tratar meu paciente (objetivo clinico). ${ }^{\mathrm{A}}$

\begin{tabular}{lccccc}
\hline Sim & $3(6,5)$ & $6(13)$ & $1(2,2)$ & $27(58,7)$ & $9(19,6)$ \\
\hline Não & 0 & $7(4,8)$ & $32(22,1)$ & $65(44,8)$ & $41(28,3)$
\end{tabular}$\quad<0,001$

\section{Fator 3. Atenção à saúde centrada no paciente}

21. Na minha profissão são necessárias habilidades de interação e cooperação com os pacientes (situação do paciente). ${ }^{\mathrm{A}}$

\begin{tabular}{lllllll}
\hline Sim & 0 & 0 & 0 & $7(15,2)$ & $39(84,8)$ & \multirow{2}{*}{0,004} \\
\hline Não & 0 & 0 & $3(2,1)$ & $58(40)$ & $84(57,9)$ &
\end{tabular}

\section{Fator 4. Competência profissional}

25. Liderança: Durante minha formação pude perceber o desenvolvimento de potencial para assumir cargos de liderança. ${ }^{\mathrm{A}}$

\begin{tabular}{lcccccc}
\hline Sim & 0 & 0 & $1(2,2)$ & $27(58,7)$ & $18(39,1)$ & \multirow{2}{*}{0,012} \\
\hline Não & 0 & $2(1,4)$ & $15(10,3)$ & $48(33,1)$ & $80(55,2)$ &
\end{tabular}

26. Administração e gerenciamento: Durante minha formação pude desenvolver aptidão para fazer gerenciamento e administração tanto da força de trabalho, dos recursos físicos e materiais e de informação. Além de aptidão para assumir cargo de gestão de equipe de saúde.

Legenda: 1 - Discordo Totalmente; 2 - Discordo; 3- Nem discordo nem concordo; 4 - Concordo; 5 - Concordo Totalmente

Tabela 1. Distribuição das respostas dos estudantes de Enfermagem nos itens da RIPLS. Sergipe, 2020. (conclusão) 


\begin{tabular}{|c|c|c|c|c|c|c|}
\hline & 1 & 2 & 3 & 4 & 5 & $\mathrm{P}$ \\
\hline Sim & 0 & $3(6,5)$ & $4(8,7)$ & $27(58,7)$ & $12(26,1)$ & \multirow{2}{*}{0,020} \\
\hline Não & 0 & $1(0,7)$ & $20(13,8)$ & $65(44,8)$ & $59(40,7)$ & \\
\hline \multicolumn{7}{|c|}{ Atua ou atuou como monitor de disciplina específica de enfermagem que possui ensino clínico X Fatores/itens RIPLS } \\
\hline \multicolumn{7}{|c|}{ Fator 1. Trabalho em equipe e colaboração } \\
\hline \multicolumn{7}{|c|}{ 11. A função dos demais profissionais da saúde é principalmente apoio aos médicos. ${ }^{\mathrm{A}}$} \\
\hline Sim & $33(78,6)$ & $7(16,6)$ & $2(4,8)$ & 0 & 0 & \multirow{2}{*}{0,007} \\
\hline Não & $70(47)$ & $54(36,2)$ & $12(8,1)$ & $9(6)$ & $4(2,7)$ & \\
\hline
\end{tabular}

Fator 2. Identidade profissional

12. Preciso adquirir muito mais conhecimento e habilidades que estudantes de outras profissões da saúde. ${ }^{\text {A }}$

\begin{tabular}{lccccccc}
\hline Sim & 0 & $20(47,6)$ & $14(33,3)$ & $8(19)$ & 0 & 0,008 \\
Não & $18(12,1)$ & $53(35,6)$ & $41(27,5)$ & $17(11,4)$ & $20(13,4)$ & 0 \\
\hline
\end{tabular}

13. Eu me sentiria desconfortável se outro estudante da área da saúde soubesse mais sobre um tópico do que eu. ${ }^{\mathrm{A}}$

\begin{tabular}{lcccccc} 
Sim & $3(7,1)$ & $27(64,3)$ & $6(14,3)$ & $4(9,5)$ & $2(4,8)$ & 0,014 \\
\hline Não & $46(30,9)$ & $63(42,3)$ & $28(18,8)$ & $9(6)$ & $3(2)$ & 0 \\
\hline
\end{tabular}

\section{Fator 4. Competência profissional}

26. Administração e gerenciamento: Durante minha formação pude desenvolver aptidão para fazer gerenciamento e administração tanto da força de trabalho, dos recursos físicos e materiais e de informação. Além de aptidão para assumir cargo de gestão de equipe de saúde. ${ }^{\text {A }}$

\begin{tabular}{lccccccc} 
Sim & 0 & 0 & $3(7,1)$ & $14(33,3)$ & $25(59,6)$ & 0,007 \\
\hline Não & 0 & $4(2,7)$ & $21(14,1)$ & $78(52,3)$ & $46(30,9)$ & 0 \\
\hline
\end{tabular}

Legenda: 1 - Discordo Totalmente; 2 - Discordo; 3- Nem discordo nem concordo; 4 - Concordo; 5 - Concordo Totalmente. Fonte: Autores (2020).

No tocante a análise fatorial relacionada ao semestre alocado dos alunos, embora mostre uma convergência na maioria das respostas ao questionário, percebe-se nas assertivas A1, A5, A7, A8, A9, A10, A11 vinculadas ao trabalho em equipe e colaboração que os alunos em períodos intermediários apresentam uma maior tendência ao trabalho em equipe (Tabela 2). Em relação a identidade profissional observa-se nas afirmativas A12 "Preciso adquirir muito mais conhecimento e habilidades que estudantes de outras profissões da saúde" e A15 "Chegar a um diagnóstico é a principal função do meu papel profissional (objetivo clínico)" divergência substancial nas respostas.

Quando questionado quanto à atenção à saúde centrada no paciente destaca-se a afirmativa "Na minha profissão são necessárias habilidades de interação e cooperação com os pacientes (situação do paciente)", com concordância expressiva nos alunos do décimo período.

Observando as assertivas A24 e A25 do fator 4, percebe-se o quanto é importante os diferentes tipos de comunicação, seja verbal ou não verbal, para a prestação de um cuidado de qualidade e para interagir com a equipe, ocupando um cargo de gestor da equipe. Referente ao fator Percepção na formação destaca-se a A30 na qual menciona e prepara do aluno para trabalhar em equipe de forma multidisciplinar e interdisciplinar, obtendo satisfação representativa do sexto ao oitavo período. Apesar da diferença estatística observa-se que os fatores 4 e 5 apresentam divergência mínima, com escore de "discordo totalmente" zerado. 
Tabela 2. Distribuição das respostas dos estudantes de Enfermagem nos itens da RIPLS. Sergipe, 2020.

\begin{tabular}{|c|c|c|c|c|c|c|}
\hline \multirow{2}{*}{$\begin{array}{l}\text { Semestre que está alocado X } \\
\text { Fatores/itens RIPLS }\end{array}$} & \multicolumn{6}{|c|}{ Porcentagem de resposta por ponto da Escala Likert } \\
\hline & 1 & 2 & 3 & 4 & 5 & $\mathrm{P}$ \\
\hline \multicolumn{7}{|c|}{ Fator 1. Trabalho em equipe e colaboração } \\
\hline \multicolumn{7}{|c|}{ 1. A aprendizagem junto com outros estudantes ajudou a me tornar um participante mais efetivo de uma equipe de saúde. ${ }^{\text {A }}$} \\
\hline 6 & 0 & 0 & 0 & $9(27,3)$ & $24(72,7)$ & \multirow{5}{*}{$<0,001$} \\
\hline 7 & 0 & 0 & $2(6,1)$ & $11(33,3)$ & $20(60,6)$ & \\
\hline 8 & 0 & 0 & 0 & $7(18,9)$ & $30(81,1)$ & \\
\hline 9 & 0 & 0 & $3(7,5)$ & $21(52,5)$ & $16(40)$ & \\
\hline 10 & 0 & 0 & $2(4,2)$ & $33(68,7)$ & $13(27,1)$ & \\
\hline \multicolumn{7}{|c|}{ 5. Para que a aprendizagem em pequenos grupos funcione, os estudantes precisam confiar e respeitar uns aos outros. ${ }^{\text {A }}$} \\
\hline 6 & 0 & 0 & 0 & $4(12,1)$ & $29(87,9)$ & \multirow{5}{*}{0,047} \\
\hline 7 & 0 & 0 & 0 & $8(24,2)$ & $25(75,8)$ & \\
\hline 8 & 0 & 0 & 0 & $2(5,4)$ & $35(94,6)$ & \\
\hline 9 & 0 & 0 & 0 & $12(30)$ & $28(70)$ & \\
\hline 10 & 0 & 0 & 0 & $8(16,7)$ & $40(83,3)$ & \\
\hline \multicolumn{7}{|c|}{$\begin{array}{l}\text { 7. Considerando minha graduação, não desperdiçaria meu tempo aprendendo junto com estudantes de outras profissões da } \\
\text { saúde. }{ }^{\text {A }}\end{array}$} \\
\hline 6 & $19(57,5)$ & $9(27,3)$ & $5(15,2)$ & 0 & 0 & \multirow{5}{*}{$<0,001$} \\
\hline 7 & $11(33,3)$ & $12(36,4)$ & $1(3)$ & $5(15,2)$ & $4(12,1)$ & \\
\hline 8 & $21(56,8)$ & $12(32,4)$ & 0 & $4(10,8)$ & 0 & \\
\hline 9 & $18(45)$ & $18(45)$ & 0 & $4(10)$ & 0 & \\
\hline 10 & $27(56,2)$ & $20(41,7)$ & 0 & $1(2,1)$ & 0 & \\
\hline \multicolumn{7}{|c|}{ 8. Habilidades para solução de problemas clínicos só devem ser aprendidas com estudantes do meu próprio curso. ${ }^{\text {A }}$} \\
\hline 6 & $17(51,5)$ & $11(33,3)$ & $5(15,2)$ & 0 & 0 & \multirow{5}{*}{0,016} \\
\hline 7 & $8(24,2)$ & $21(63,6)$ & 0 & $2(6,1)$ & $2(6,1)$ & \\
\hline 8 & $20(54,1)$ & $13(35,1)$ & $1(2,7)$ & 0 & $3(8,1)$ & \\
\hline 9 & $18(45)$ & $18(45)$ & $2(5)$ & $1(2,5)$ & $1(2,5)$ & \\
\hline 10 & $18(37,5)$ & $27(56,3)$ & $3(6,3)$ & 0 & 0 & \\
\hline \multicolumn{7}{|c|}{$\begin{array}{l}\text { 9. Gostaria de ter a oportunidade de trabalhar em projetos, em pequenos grupos, com estudantes de outras profissões da } \\
\text { saúde. A }\end{array}$} \\
\hline 6 & 0 & 0 & $4(12,1)$ & $7(21,2)$ & $22(66,7)$ & \multirow{5}{*}{0,015} \\
\hline 7 & 0 & 0 & $1(3)$ & $16(48,5)$ & $16(48,5)$ & \\
\hline 8 & 0 & 0 & 0 & $14(37,8)$ & $23(62,2)$ & \\
\hline 9 & 0 & 0 & $1(2,5)$ & $21(52,5)$ & $18(45)$ & \\
\hline 10 & 0 & 0 & 0 & $24(50)$ & $24(50)$ & \\
\hline \multicolumn{7}{|c|}{$\begin{array}{l}\text { 10. A aprendizagem compartilhada durante a graduação contribuiu para tornar-me um profissional que trabalha melhor em } \\
\text { equipe. }{ }^{\text {A }}\end{array}$} \\
\hline 6 & 0 & 0 & 0 & $3(9,1)$ & $30(90,9)$ & \multirow{5}{*}{0,002} \\
\hline 7 & 0 & 0 & $2(6,0)$ & $5(15,2)$ & $26(78,8)$ & \\
\hline 8 & 0 & 0 & $1(2,7)$ & $19(51,4)$ & $17(45,9)$ & \\
\hline 9 & 0 & 0 & 0 & $15(37,5)$ & $25(62,5)$ & \\
\hline 10 & 0 & 0 & $2(4,2)$ & $13(27,1)$ & $33(68,8)$ & \\
\hline
\end{tabular}

11. A função dos demais profissionais da saúde é principalmente apoio aos médicos. ${ }^{\mathrm{A}}$

\begin{tabular}{lllllll}
\hline 6 & $23(69,7)$ & $8(24,2)$ & $2(6,1)$ & 0 & 0 & $<0,001$ \\
\hline 7 & $13(39,4)$ & $13(39,4)$ & $2(6,0)$ & $3(9,1)$ & $2(6,1)$ & \\
\hline
\end{tabular}

Legenda: 1 - Discordo Totalmente; 2 - Discordo; 3 - Nem discordo nem concordo; 4 - Concordo; 5 - Concordo Totalmente 
Tabela 2. Distribuição das respostas dos estudantes de Enfermagem nos itens da RIPLS. Sergipe, 2020. (Continuação)

\begin{tabular}{lcccccc} 
Semestre que está alocado $\mathbf{X}$ & \multicolumn{4}{c}{ Porcentagem de resposta por ponto da Escala Likert } & & P \\
\cline { 2 - 6 } & Fatores/itens RIPLS & 1 & 2 & 3 & 4 & 5 \\
\hline 8 & $27(73)$ & $3(8,1)$ & $2(5,4)$ & $3(8,1)$ & $2(5,4)$ \\
\hline 9 & $11(27,5)$ & $23(57,5)$ & $3(7,5)$ & $3(7,5)$ & 0 \\
\hline 10 & $29(60,4)$ & $14(29,2)$ & $5(10,4)$ & 0 & 0 \\
\hline
\end{tabular}

Fator 2. Identidade profissional

12. Preciso adquirir muito mais conhecimento e habilidades que estudantes de outras profissões da saúde. ${ }^{\mathrm{A}}$

\begin{tabular}{lcccccc}
\hline 6 & $4(12,1)$ & $10(30,3)$ & $11(33,3)$ & $5(15,2)$ & $3(9,1)$ \\
\hline 7 & $3(9,1)$ & $5(15,2)$ & $15(45,5)$ & $5(15,2)$ & $5(15,2)$ \\
\hline 8 & $7(18,9)$ & $19(51,4)$ & $8(21,6)$ & $3(8,1)$ & 0 & 0,015 \\
\hline 10 & $2(5)$ & $21(52,5)$ & $9(22,5)$ & $2(5)$ & $6(15)$ \\
\hline
\end{tabular}

15. Chegar a um diagnóstico é a principal função do meu papel profissional (objetivo clínico). ${ }^{\text {A }}$

\begin{tabular}{lccccc}
\hline 6 & $7(21,2)$ & $9(27,3)$ & $8(24,2)$ & $7(21,2)$ & $2(6,1)$ \\
\hline 7 & $1(3)$ & $12(36,4)$ & $11(33,3)$ & $7(21,2)$ & $2(6,1)$ \\
\hline 8 & $3(8,1)$ & $5(13,5)$ & $13(35,1)$ & $11(29,7)$ & $5(13,5)$ \\
\hline 9 & $2(5)$ & $19(47,5)$ & $8(20)$ & $5(12,5)$ & $6(15)$ \\
\hline 10 & $4(8,3)$ & $23(47,9)$ & $9(18,8)$ & $6(12,5)$ & $6(12,5)$
\end{tabular}

0,039

Fator 3. Atenção à saúde centrada no paciente

21. Na minha profissão são necessárias habilidades de interação e cooperação com os pacientes (situação do paciente). ${ }^{\mathrm{A}}$

\begin{tabular}{lllcccc}
\hline 6 & 0 & 0 & $2(6,0)$ & $6(18,2)$ & $25(75,8)$ \\
\hline 7 & 0 & 0 & 0 & $9(27,3)$ & $24(72,7)$ \\
\hline 8 & 0 & 0 & 0 & $8(21,6)$ & $29(78,4)$ & 0,007 \\
\hline 9 & 0 & 0 & $1(2,5)$ & $17(42,5)$ & $22(55)$ \\
\hline 10 & 0 & 0 & 0 & $25(52,1)$ & $23(47,9)$
\end{tabular}

\section{Fator 4. Competência profissional}

24. Comunicação: Durante minha formação pude perceber desenvolvimento de efetividade na comunicação verbal, não verbal e nas habilidades de escrita e leitura, para comunicação com outros profissionais e pessoas envolvidas com meus cuidados. $^{\mathrm{A}}$

\begin{tabular}{llcccl}
\hline 6 & 0 & 0 & 0 & $12(36,4)$ & $21(63,6)$ \\
\hline 7 & 0 & $3(9,1)$ & 0 & $11(33,3)$ & $19(57,6)$ \\
\hline 8 & 0 & 0 & $1(2,7)$ & $13(35,1)$ & $23(62,2)$ \\
\hline 9 & 0 & 0 & $1(2,5)$ & $20(50)$ & $19(47,5)$ \\
\hline 10 & 0 & 0 & $2(4,2)$ & $32(66,6)$ & $14(29,2)$ \\
\hline
\end{tabular}

26. Administração e gerenciamento: Durante minha formação pude desenvolver aptidão para fazer gerenciamento e administração tanto da força de trabalho, dos recursos físicos e materiais e de informação. Além de aptidão para assumir cargo de gestão de equipe de saúde. ${ }^{\mathrm{A}}$

\begin{tabular}{llllll}
\hline 6 & 0 & 0 & $5(15,2)$ & $20(60,6)$ & $8(24,2)$ \\
\hline 7 & 0 & 0 & $4(12,1)$ & $19(57,6)$ & $10(30,3)$ \\
\hline 8 & 0 & 0 & $8(21,6)$ & $4(10,8)$ & $25(67,6)$ \\
\hline 9 & 0 & 0 & $4(10)$ & $21(52,5)$ & $15(37,5)$ \\
\hline 10 & 0 & $4(8,3)$ & $3(6,3)$ & $28(58,3)$ & $13(27,1)$ \\
\hline
\end{tabular}

Fator 5. Percepção na formação

30. Foi desenvolvido em minha formação capacidade para trabalho em equipe multiprofissional e interdisciplinar. ${ }^{\mathrm{A}}$

\begin{tabular}{|c|c|c|c|c|c|c|}
\hline 6 & 0 & $1(3)$ & $4(12,1)$ & $11(33,3)$ & $17(51,6)$ & $<0,001$ \\
\hline 7 & 0 & $4(12,1)$ & $1(3)$ & $12(36,4)$ & $16(48,5)$ & \\
\hline
\end{tabular}

Legenda: 1 - Discordo Totalmente; 2 - Discordo; 3- Nem discordo nem concordo; 4 - Concordo; 5 - Concordo Totalmente

Tabela 2. Distribuição das respostas dos estudantes de Enfermagem nos itens da RIPLS. Sergipe, 2020. (Conclusão) 


\begin{tabular}{|c|c|c|c|c|c|c|}
\hline Fatores/itens RIPLS & 1 & 2 & 3 & 4 & 5 & $\mathrm{P}$ \\
\hline 8 & 0 & $1(2,7)$ & $5(13,5)$ & $4(10,8)$ & $27(73)$ & \\
\hline 9 & 0 & $1(2,5)$ & $5(12,5)$ & $17(42,5)$ & $17(42,5)$ & \\
\hline 10 & 0 & $2(4,2)$ & $1(2,1)$ & $33(68,7)$ & $12(25)$ & \\
\hline
\end{tabular}

Legenda: 1 - Discordo Totalmente; 2 - Discordo; 3- Nem discordo nem concordo; 4 - Concordo; 5 - Concordo Totalmente. Fonte: Autores (2020).

\section{Discussão}

A formação para o cuidado integral e trabalho em equipe são reconhecidas no cenário mundial como elementos da qualidade da formação profissional e da atenção à saúde. No contexto dessa pesquisa, o questionário buscou alcançar a perspectiva quanto a integralidade do cuidado e o trabalho em equipe, avaliada a partir da experiência vivenciada na graduação, tal como as práticas clínicas, monitorias acadêmicas e atividades adicionais como os estágios extracurriculares.

A partir dos dados sociodemográficos, observou-se que os participantes deste estudo eram predominantemente sexo feminino, fato que corrobora com outras pesquisas realizadas com acadêmicos de enfermagem (Souza et al., 2019; Spagnol et al., 2017). A representatividade feminina na área está associada a fatores históricos e ao nome de grandes mulheres, pioneiras na profissão que transformaram as práticas do cuidado, elevaram a assistência prestada, contribuindo para o fortalecimento e reconhecimento da classe (Somariva et al., 2019).

Em relação às atividades extraclasses desenvolvidas pelos discentes no período da graduação, foram analisadas a realização de monitoria acadêmica de disciplinas com saberes específicos do curso e estágio extracurricular com prática assistencial. Apesar da minoria dos alunos fazerem menção a essas atividades, os resultados da pesquisa apontam olhares diferentes e entendimento da importância do trabalho em equipe, colaborações e identidade profissionais mais consolidados quando comparados com alunos que não tiveram essas vivências.

Pesquisas recentes deixam claras as contribuições dessas práticas no processo de aprendizagem, tal como, no desenvolvimento de habilidades e competências, além de contribuir com a formação crítica, criativa e resolutiva, direcionada ao atendimento de diversas demandas atreladas ao mercado de trabalho (Puschel et al., 2017; Barros et al., 2020).

Diante das demandas do mundo do trabalho a assistência do enfermeiro precisa estar respaldada em ciência e ter como foco o cuidado integral e efetivo nos diferentes níveis de atenção à saúde e para alcançar essa efetividade precisam ter conhecimento dos processos individuais e coletivos, associados ao desempenho eficaz da equipe interprofissional (Carvalho et al., 2020).

No tocante a atenção centrada do paciente, os alunos concordam que além de interagir com a equipe multiprofissional, são necessárias habilidades de interação e cooperação com os pacientes. Estudo realizado em 2019 apontam resultados parecidos com a pesquisa atual, no qual, os autores justificam que o principal foco da enfermagem é o cuidado embasado na ciência e em características humanísticas (Blasa et al., 2019). Em contrapartida, a pesquisa de Garcia e colaboradores (2020) mostra que os discentes separam as ações assistenciais e gerenciais, o que dificulta uma totalidade e direcionamento de cuidado integral. Dessa forma, tem-se como justificativa que a enfermagem ainda se apresenta hospitalocêntrica, focada na cura da doença, apenas.

Considerando todos os alunos envolvidos na pesquisa apesar dos resultados indicarem que os discentes percebem a importância das habilidades de trabalho em equipe no processo de aprendizagem e ressaltar uma relação de confiança e respeito mútuo, percebe-se divergência quando concordam que o processo de aprendizagem e desenvolvimento de habilidades clínicas não devem ser aprendidas com estudantes de cursos diferentes.

Scofano, Lanzillotti e Valente (2019) admitem que para o enfermeiro, trabalhar em equipe, colocando em prática a liderança, torna-se uma atividade complexa, porém de extrema necessidade. A troca de conhecimento faz-se essencial na 
prestação de cuidado pois trata-se de um objetivo comum: a assistência. Os profissionais devem interagir entre si, valorizando as diferentes áreas de atuação e núcleo de saber de cada uma.

Nesse contexto, algumas IES associam as suas práticas pedagógicas a interdisciplinaridade almejando integrar, articular e estimular o trabalhar em conjunto, proporcionando aos discentes a troca de saberes e uma aproximação da realidade profissional no cotidiano das áreas da saúde. Para Rissi e Higarashi (2017), por exemplo, o uso de simulações realísticas tornou-se instrumento essencial no processo de aprendizagem para os futuros profissionais.

Dando continuidade ao pensamento das autoras supracitadas, essa metodologia de ensino além de permitir que o estudante se aproxime ao máximo do contexto vivido no cotidiano, oportuniza o discente de interagir com a equipe multidisciplinar nas diversas situações que surgem. Colabora também para estimular o aluno a trabalhar competências como liderança e comunicação com os diferentes profissionais.

No que diz respeito às competências desenvolvidas durante a graduação, observou-se predomínio de respostas que estão de acordo com o estabelecido nas Diretrizes Curriculares Nacionais do curso de Enfermagem e com a formação para o SUS. Em análise as respostas dos discentes em comparação ao andamento da graduação constatou-se que o nível de concordância foi representativo nos alunos dos últimos períodos. O resultado deve-se ao fato de os alunos concludentes já terem uma boa experiência com a prática clínica possuindo uma visão ampla sobre o tema. A pesquisa de Rodrigues e colaboradores (2019) apresenta resultados semelhantes aos dessa pesquisa, onde os concluintes do curso relatam que os estágios contribuíram para a sua formação profissional e para o aprimoramento de suas práticas atendendo as expectativas.

No tocante percepção e formação, foi observado que as respostas se apresentaram divergentes, sendo que, a maioria concorda com a assertiva apresentada. O curso em análise proporciona aos alunos durante o seu período de formação oportunidades de trabalhar em equipe. Dessa forma, os discentes passam a compreender que é necessário trabalhar em equipe para proporcionar melhor resolutividade das ações.

A pesquisa de Carvalho, Junior e Siqueira (2019) refere que o ensino da graduação apresenta-se centrado na em um único aspecto, o que dificulta o desenvolvimento de novas formas de aprendizagem e conhecimento. Porém outros autores demostraram resultados diferentes, onde os alunos relatam que as práticas integrativas realizadas durante o período de formação forneceram o aprimoramento da habilidade de trabalhar em equipe além do desenvolvimento de outras habilidades (2017).

Segundo Camarori e outros autores (2020) as transformações que ocorreram na educação ao longo do tempo faz com que os currículos sejam modificados e reinventados. A nova forma de ensinar faz com seja desenvolvida a prática do trabalho em equipe a autonomia e a interdisciplinaridade, tornando o aluno protagonista da ação facilitando a comunicação e posteriormente o destaque e a atuação no mercado de trabalho.

\section{Considerações Finais}

Embora tenham sido feitas todas as tentativas para aderir a estruturas metodológicas e critérios de avaliação estabelecidos, a pesquisa apresenta limitações que afetam a aplicabilidade das conclusões tiradas e a generalização dos resultados. $\mathrm{O}$ uso do questionário em formato online pode ter comprometido o alcance a população estudada resultando a um número de respostas inferior a $50 \%$.

Independente de suas limitações, esta pesquisa faz algumas contribuições importantes. Este trabalho contribui e possibilita reflexões quanto a melhorias da assistência na perspectiva do cuidado integral e trabalho em equipe alcançada por meio do processo de formação e realização de novas pesquisas com foco na prática profissional. 
Observa-se evidentemente o quanto que os discentes compreendem a importância do tema e a necessidade de trabalhá-lo ainda na formação do profissional enfermeiro. O estudante deve procurar desenvolver as competências e habilidades ainda na graduação, para que ao ingressar no mercado de trabalho, utilize-as devidamente.

Os novos enfermeiros devem liderar sua equipe e interagir bem com a mesma, valorizando as diferentes classes e profissões, ressaltando a importância de cada uma para a prestação de um cuidado integral. Dessa forma, cada paciente é atendido de forma individualizada, por uma equipe multiprofissional sincronizada, prestando uma assistência de qualidade.

É essencial que as universidades que ofertam um ensino superior de enfermagem insiram em suas matrizes curriculares múltiplas atividades que estimulem o discente a trabalhar em equipe e a olhar o paciente de forma holística, oferendo uma assistência ideal.

\section{Referências}

Azevedo, C., Moura, C. D. C., Corrêa, H. P., Mata, L. R. F. D., Chaves, É. D. C. L., \& Chianca, T. C. M. (2019). Práticas integrativas e complementares no âmbito da enfermagem: aspectos legais e panorama acadêmico-assistencial. Escola Anna Nery, 23(2). http://www.scielo.br/pdf/ean/v23n2/pt_1414-8145-ean23-02-e20180389.pdf.

Barbosa, A. C. S., Luiz, F. S., Friedrich, D. B. D. C., Püschel, V. A. D. A., Farah, B. F., \& Carbogim, F. D. C. (2019). Profile of nursing graduates: competencies and professional insertion. Revista Latino-Americana de Enfermagem, 27. http://www.scielo.br/pdf/rlae/v27/pt_0104-1169-rlae-27-e3205.pdf.

Barros, A. W. M. S., Araújo, D. L., de Barros Lima, M. B. R., de Albuquerque, E. A., de Melo, J. F., de Couto Calado, E. L., ... \& Marinho, E. R. S. (2020). Monitoria acadêmica em enfermagem: uma revisão de literatura/Academic monitoring in nursing: a literature review. Brazilian Journal of Health Review, 3(3), 4785-4794. DOI: 10.34119/bjhrv3n3-067.

Barros, E. R. S., \& Ellery, A. E. L. Colaboração interprofissional em uma unidade de terapia intensiva: desafios e possibilidades. Revista da Rede de Enfermagem do Nordeste, 17(1), 10-19. http://www.periodicos.ufc.br/rene/article/view/2600/1989

Caramori, U., Mello, J. B. D., Barretto, C. A. P., Costa, R. D. M. R., Peña, S. S., Ramos, A. L. C., \& Pavan, M. V. (2020). Projeto FELLOWS: habilidades de educação para estudantes das profissões da saúde. Revista Brasileira de Educação Médica, 44(1). http://doi.org/10.1590/1981-527v44.1-20190233.

Carvalho, D. P. D. S. R. P., Vitor, A. F., Cogo, A. L. P., Bittencourt, G. K. G. D., Santos, V. E. P., \& Ferreira Júnior, M. A. (2020). Mensuração do pensamento crítico geral em estudantes de cursos de graduação em enfermagem: estudo experimental. Texto \& Contexto-Enfermagem, 29. http://dx.doi.org/10.1590/1980-265X-TCE-2018-0229

Carvalho, V. C. D. S., Junior, A. C. S., \& Siqueira, F. P. C. (2019). Trabalho em grupo: a percepção do profissional do sistema único saúde. Investigación en Enfermería: Imagen y Desarrollo, 21(1). https://pesquisa.bvsalud.org/portal/resource/pt/biblio-995568.

Chaves, L. D. P., Mininel, V. A., Silva, J. A. M. D., Alves, L. R., Silva, M. F. D., \& Camelo, S. H. H. (2017). Nursing supervision for care comprehensiveness. Revista Brasileira de Enfermagem, 70(5), 1106-1111. http://www.scielo.br/pdf/reben/v70n5/pt_0034-7167-reben-70-05-1106.pdf.

Deane, W. H., \& Fain, J. A. (2016). Incorporating Peplau's theory of interpersonal relations to promote holistic communication between older adults and nursing students. Journal of Holistic Nursing, 34(1), 35-41. https://doi.org/10.1177/0898010115577975.

Duarte, M. D. L. C., \& Boeck, J. N. (2015). O trabalho em equipe na enfermagem e os limites e possibilidades da estratégia saúde da família. Trabalho, Educação e Saúde, 13(3), 709-720. http://dx.doi.org/10.1590/1981-7746-sip00054.

Garcia, F. R., Rendón, D. D. C. S., Nazareth, J. B., Amorim, T. V., Arreguy-Sena, C., \& Salimena, A. M. D. O. (2020). Sentidos do cuidado para acadêmicos de enfermagem: contribuições heideggerianas para o ensino. Rev. Pesqui. (Univ. Fed. Estado Rio J., Online), 318-323. https://pesquisa.bvsalud.org/portal/resource/pt/biblio-1051232.

García-Carpintero Blas, E., Siles-González, J., Martínez-Roche, M. E., Martínez-Miguel, E., Manso-Perea, C., González-Cervantes, S., \& García-García, E. (2019). Percepciones de los estudiantes sobre sus vivencias en las prácticas clínicas. Enfermería universitaria, 16(3), 259-268. http://ciberindex.com/index.php/reu/article/view/259268reu.

Negri, E. C., Mazzo, A., Martins, J. C. A., Pereira Junior, G. A., Almeida, R. G. D. S., \& Pedersoli, C. E. (2017). Clinical simulation with dramatization: gains perceived by students and health professionals. Revista latino-americana de enfermagem, 25. https://doi.org/10.1590/1518-8345.1807.2916

Peduzzi, M., Agreli, H. L. F., Silva, J. A. M. D., \& Souza, H. S. D. (2020). Trabalho em equipe: uma revisita ao conceito e a seus desdobramentos no trabalho interprofissional. Trabalho, Educação e Saúde, 18. http://www.scielo.br/pdf/tes/v18s1/1678-1007-tes-18-s1-e0024678.pdf.

Peduzzi, M., Norman, I. J., Germani, A. C. C. G., Silva, J. A. M. D., \& Souza, G. C. D. (2013). Educação interprofissional: formação de profissionais de saúde para o trabalho em equipe com foco nos usuários. Revista da Escola de Enfermagem da USP, 47(4), 977-983. https://doi.org/10.1590/S0080623420130000400029

Pereira, A. S., Shitsuka, D. M., Parreira, F. B., \& Shitsuka, R. (2018). Metodologia da pesquisa científica [recurso eletrônico [eBook]. Santa Maria. Ed. UAB/NTE/UFSM. https://repositorio. ufsm. br/bitstream/handle/1/15824/Lic_Computacao_MetodologiaPesquisa-Cientifica. pdf.

Interpersonales, T. D. L. R., Terapéutica, R. A. D. L. F., \& Enfermero, D. (2019). Teoria das relações interpessoais: reflexões acerca da função terapêutica do enfermeiro em saúde mental. 
Research, Society and Development, v. 10, n. 1, e24110111724, 2021

(CC BY 4.0) | ISSN 2525-3409 | DOI: http://dx.doi.org/10.33448/rsd-v10i1.11724

Püschel, V. A. A., Costa, D., Reis, P. P., Oliveira, L. B., \& Carbogim, F. C. (2017). The nurse in the job market: insertion, skills and abilities. Rev Bras Enferm, 70(6), 1288-1295. http://dx.doi.org/10.1590/0034-7167-2016-0061.

Reuter, C. L. O., Santos, V. C. F. D., \& Ramos, A. R. (2018). The exercise of interprofessionality and intersetoriality as an art of caring: innovations and challenges. Escola Anna Nery, 22(4). https://www.scielo.br/scielo.php?script=sci_arttext\&pid=S1414-81452018000400221.

Rissi, G. P., Shibukawa, B. M. C., de Lima, M. F., \& Higarashi, I. H. (2020). Simulação clínica de emergência infantil no contexto da formação profissional em enfermagem: revisão integrativa. Research, Society and Development, 9(7), e421974261-e421974261.

Rodrigues, M. N. D. A., Santos, R. B. D., Silva, F. F. D., Sardinha, D. M., Prazeres, P. D. S. C. D., \& Silva, A. G. I. D. (2019). O estágio curricular supervisionado em enfermagem sob a ótica dos concluintes do curso. Nursing (Säo Paulo), 3280-3285. http://pesquisa.bvsalud.org/org/portal/resource/pt/biblio-1051579.

Santos, A. T. S., Oliveira, C. B., Passos, M. C., Andrade, A. S. A., \& Gallotti, F. C. M. (2019). Integralidade do cuidado na formação do enfermeiro: visões e vivências do acadêmico de enfermagem. Enfermagem em Foco. 10(1). http://biblioteca.cofen.gov.br/wpcontent/uploads/2020/04/Integralidadedocuidadonaformacaodoenfermeiro.pdf

Scofano, B. D. S., Valente, G. S. C., \& Lanzillotti, R. S. (2019). Atuação do enfermeiro enquanto líder de equipe na área hospitalar: uma revisão integrativa. Nursing (Säo Paulo), 2943-2948. https://pesquisa.bvsalud.org/portal/resource/pt/biblio-1025548.

Somariva, V. C. A., Birolo, I. V. B., Tomasi, C. D., \& Soratto, J. Percepção das equipes de enfermagem das equipes de enfermagem na atenção básica frente à sistematização da assistência de enfermagem. (2019). Enferm Foco. 10 (4): 142-147. http://revista.cofen.gov.br/index.php/enfermagem/article/view/2221

Souza, W., Mayara, I., Mayk, A., Maynard, T., Silva, G., \& Lira, A. Percepção dos profissionais de enfermagem sobre o seu conselho de classe. (2019). Revista Enfermagem em Foco. 10(6). http://revista.cofen.gov.br/index.php/enfermagem/article/view/1952

Spagnol, G. S., Vergílio, M. S. T. G., Bergamasco, J. G. P., \& Silva, E. M. Arte e ação: iluminando novos caminhos para a enfermagem. (2017). História da Enfermagem Revista Eletrônica. 8(2). http://here.abennacional.org.br/here/v8/n2/a02.pdf. 